Article

\title{
Effect of Different Manufacturing Methods on the Conflict between Porosity and Mechanical Properties of Spiral and Porous Polyethylene Terephthalate/Sodium Alginate Bone Scaffolds
}

\author{
Ching-Wen Lou ${ }^{1}$, Chien-Lin Huang ${ }^{2}$, Chih-Kuang Chen ${ }^{3}$, Chi-Fan Liu ${ }^{4}$, Shih-Peng Wen ${ }^{5}$ \\ and Jia-Horng Lin $5,6,7, *$
}

Received: 16 September 2015; Accepted: 7 December 2015; Published: 14 December 2015

Academic Editor: Wen-Hsiang Hsieh

1 Institute of Biomedical Engineering and Materials Science, Central Taiwan University of Science and Technology, Taichung 40601, Taiwan; cwlou@ctust.edu.tw

2 Department of Fiber and Composite Materials, Feng Chia University, Taichung City 40724, Taiwan; clhuang@mail.fcu.edu.tw

3 The Polymeric Biomaterials Lab, Department of Fiber and Composite Materials, Feng Chia University, Taichung City 40724, Taiwan; chihkchen@fcu.edu.tw

4 Office of Physical Education and Sports Affairs, Feng Chia University, Taichung 40724, Taiwan; cfliu@fcuoa.fcu.edu.tw

5 Laboratory of Fiber Application and Manufacturing, Department of Fiber and Composite Materials, Feng Chia University, Taichung City 40724, Taiwan; waz770207@gmail.com

6 School of Chinese Medicine, China Medical University, Taichung 40402, Taiwan

7 Department of Fashion Design, Asia University, Taichung 41354, Taiwan

* Correspondence: jhlin@fcu.edu.tw; Tel.: +886-4-2451-7250 (ext. 3405)

\begin{abstract}
In order to solve the incompatibility between high porosity and mechanical properties, this study fabricates bone scaffolds by combining braids and sodium alginate (SA) membranes. Polyethylene terephthalate (PET) plied yarns are braided into hollow, porous three dimensional (3D) PET braids, which are then immersed in SA solution, followed by cross-linking with calcium chloride $\left(\mathrm{CaCl}_{2}\right)$ and drying, to form PET bone scaffolds. Next, SA membranes are rolled and then inserted into the braids to form the spiral and porous PET/SA bone scaffolds. Samples are finally evaluated for surface observation, porosity, water contact angle, compressive strength, and MTT assay. The test results show that the PET bone scaffolds and PET/SA bone scaffolds both have good hydrophilicity. An increasing number of layers and an increasing $\mathrm{CaCl}_{2}$ concentration cause the messy, loose surface structure to become neat and compact, which, in turn, decreases the porosity and increases the compressive strength. The MTT assay results show that the cell viability of differing SA membranes is beyond 100\%, indicating that the PET/SA bone scaffolds containing SA membranes are biocompatible for cell attachment and proliferation.
\end{abstract}

Keywords: plied yarns; braid; sodium alginate (SA); bone scaffold

\section{Introduction}

Tissue engineering, a field comprising different sciences, emphasizes the development of the bio-substitute as well as repair, maintenance of, or improvement on the functions of tissues [1]. Bone tissue engineering is important for the impaired bones, and bone scaffolds can provide the bone tissues with a sufficient pore size and porosity for their infiltration, so as to serve as a carrier for osteoblasts for the repair and treatment. Polymers can serve as a supportive façade, facilitating the 
stabilization of bioactive molecules and cell attachment. As a result, polymers have been commonly used for tissue substitution, tissue reinforcement, and organ transplantation [2].

Non-degradable synthesis polymers, such as polyethyle (PE), nylon, and polyethylene terephthalate (PET), have been clinically applied as artificial ligaments and artificial blood vessels [3] due to their stability, flexibility, and durability in the human body. In particular, PET possesses excellent properties, like high specific strength and high anti-corrosion, and has a low production cost. However, PET has a low surface energy, which results in a low hydrophilicity and attachment [2] that influence the infiltration of tissues. Therefore, the combination of natural polymers like sodium alginate (SA), chitin, and chitosan that augment the osteogenetic property can help with the replacement, repair, and reconstruction of the impaired bones [4]. SA is a polysaccharide that is primarily derived from brown algae and is pervasively used in the production of biomedical hydrogel, and has biocompatibility, a low production cost, and does not have toxicity. Furthermore, SA transforms into hydrogel as a result of the combination of bivalent cations (e.g., calcium cations and barium ions) [5], and a cross-linking with $\mathrm{CaCl}_{2}$ can prevent the hydrogel from dissolving in solution, such as a medium, which enables its application in three dimensional (3D) bone scaffolds [2].

The bone scaffolds used in bone tissue engineering are required to have (a) biocompatibility, which allows their performance in the host without causing any immune responses; (b) an interconnected pore structure, which contributes to an even distribution of stress over the entire bone scaffold, a high porosity and a large specific area that allow tissues to grow inwardly, angiogenesis, and the formation of bones, capillary chemistry, and osteoinductivity and (c) mechanical properties, which provide the impaired area with temporary support and bear the interior load of the body. Additionally, the clinical use and ease of preparation should also be considered. However, some studies pointed out that with a greater porosity and a large size of pores, the bone scaffolds have lower mechanical properties [6]. Therefore, this study combines PET braids and SA membranes in order to yield sufficient mechanical properties, a high porosity, and a three-dimensional structure, which can fortify the repair ability of the impaired bones.

In order to solve the conflict between mechanical properties and a high porosity, this study uses a 16-spindle braider for a multi-layer braiding of PET plied yarns, forming 3D and porous PET braids. The loose structure of the PET braids is then stabilized as a result of the immersion in SA solution and being dried, forming PET bone scaffolds. Afterwards, the SA membranes that have been cross-linked with $\mathrm{CaCl}_{2}$ solution are rolled and then inserted into the stabilized PET braids to form PET/SA bone scaffolds, the mechanical properties and porosity of which are finally evaluated. The structural design proposed by this study can provide the outer layer with porosity, which facilitates the transmission of nutrition and impurities and mechanical properties that can support the whole structure, while the interlayer of SA membranes can help with cell attachment for tissue growth.

\section{Results and Discussion}

\subsection{Surface Observation of PET Bone Scaffolds: Effect of Number of Layers and Concentration of $\mathrm{CaCl}_{2}$ Solution on the Fiber Arrangement}

Figure 1 shows that the PET bone scaffolds multi-braided with 15 or 20 layers exhibit a messy surficial structure regarding the concentrations of the $\mathrm{CaCl}_{2}$ solution. Because the braiding structure is loose, fibers are arranged disorderly. By contrast, the fibers are arranged orderly as a result of the compact structure caused by the increasing number of layers. 

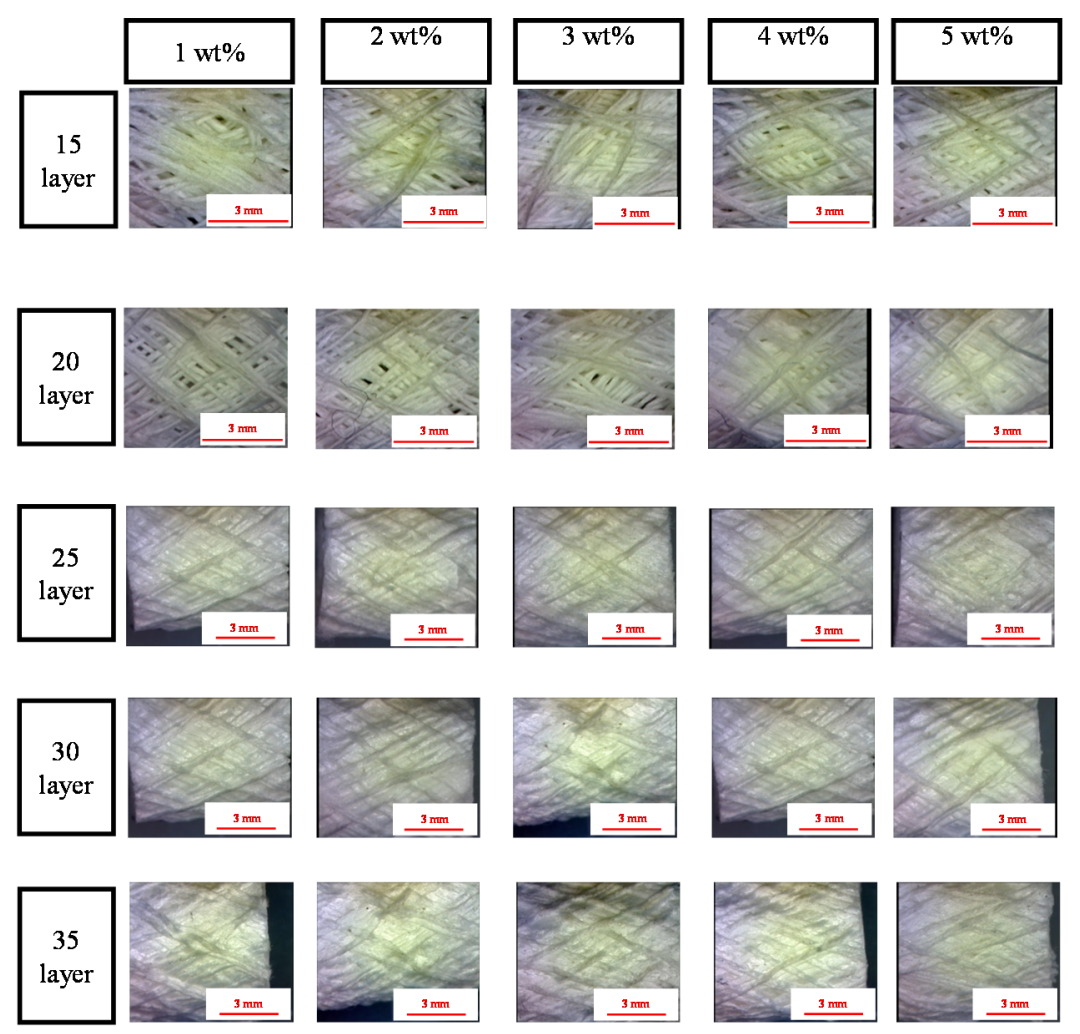

Figure 1. Morphology of the polyethylene terephthalate (PET) bone scaffolds that are immersed in a $3 \mathrm{wt} \%$ sodium alginate (SA) solution as related to various cross-linking concentrations and differing numbers of layers. The scale bar is $3 \mathrm{~mm}$.

\subsection{Effect of Number of Layers and Concentration of $\mathrm{CaCl}_{2}$ Solution on the Porosity of PET Bone Scaffolds}

Figure 2 shows that the porosity of the PET bone scaffolds is between $80 \%$ and $90 \%$ regardless of the number of layers and the concentration of the $\mathrm{CaCl}_{2}$ solution. Due to a multi-layer braiding which provides the interconnected pores between layers, PET bone scaffolds have a high porosity; however, the porosity slightly decreases when the number of layers increases. When braided with no less than 25 layers, the porosity of the PET bone scaffolds decreases because the pores of their surface disappear as a result of the orderly-arranged braiding structure.

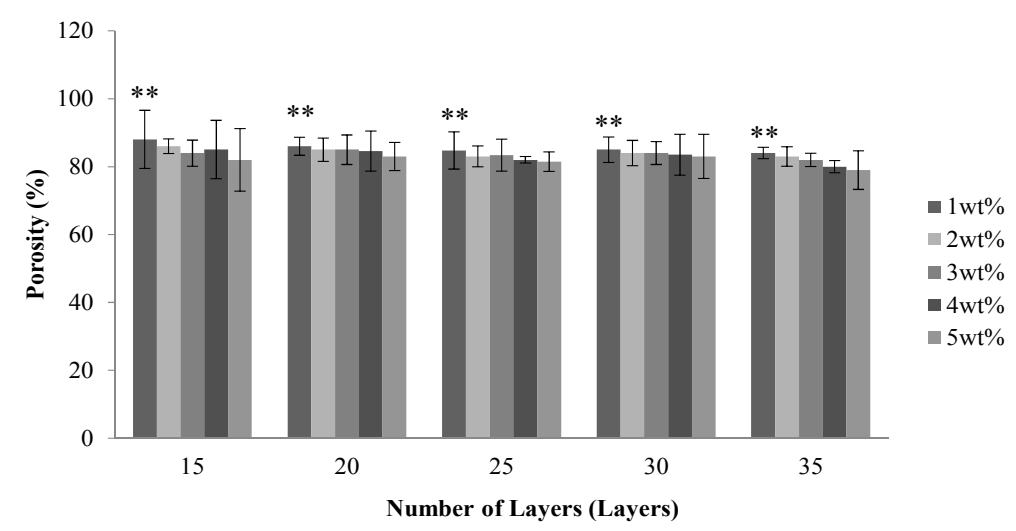

Figure 2. Porosity of the PET bone scaffolds as related to various numbers of layers and various concentrations of $\mathrm{CaCl}_{2}$ solution. ${ }^{* *} p<0.01$. 
With a specified number of layers, PET bone scaffolds that are cross-linked with 1 wt $\% \mathrm{CaCl}_{2}$ solution have a greater porosity. A low concentration results in a low cross-linking level with SA. Therefore, the PET filaments are loosely arranged, which, in turn, creates more space between them and heightens the porosity. The porosity decreases when the PET bone scaffolds cross-link with an increasing concentration of $\mathrm{CaCl}_{2}$ solution, which results in a greater cross-linking level of SA and subsequently causes the fibers to be arranged more tightly.

\subsection{Effect of Number of Layers and Concentration of $\mathrm{CaCl}_{2}$ Solution on the Hydrophilicity of PET Bone Scaffolds}

Figure 3 shows that the water contact angle increases as a result of increasing number of layers, and the water contact angle is between $6^{\circ}$ and $18^{\circ}$. Although PET filaments are hydrophobic, the PET multi-layer braids have a micro-pore structure over their surface, which can augment the hydrophilicity of the surface and the water infiltration, and thus the water molecules enter the interior of PET filaments. Such a result causes a very low surface tension between the PET bone scaffolds and water; therefore, the water contact angle is small.

With a specified number of layers, the water contact angle of the PET bone scaffolds is proportional to the concentration of $\mathrm{CaCl}_{2}$ solution, as seen in Figure 3. When cross-linking with $1 \mathrm{wt} \% \mathrm{CaCl}_{2}$ solution, the PET filaments are loosely arranged due to the low cross-linking level, which enables water molecules to easily infiltrate the interior, and thus the water contact angle is small. The cross-linking level increases with the increasing concentration of $\mathrm{CaCl}_{2}$ solution, which results in a compact arrangement of PET filaments. The micro-pore structure over the surface disappears, which prevents the water molecules from entering the interior of the bone scaffolds, and eventually results in a greater water contact angle.

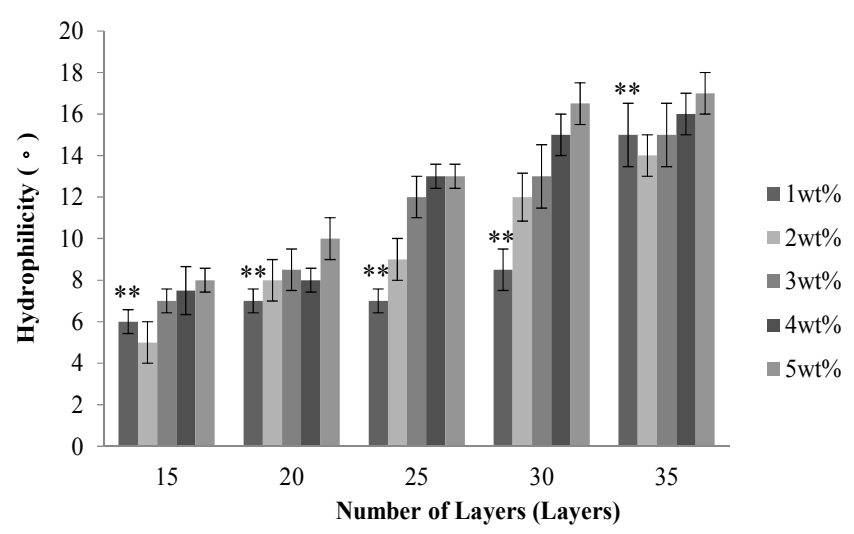

Figure 3. Water contact angle of the PET bone scaffolds composed of various numbers of layers and cross-linked with different concentrations of $\mathrm{CaCl}_{2}$ solution. ${ }^{* *} p<0.01$.

\subsection{Effect of Number of Layers and Concentration of $\mathrm{CaCl}_{2}$ Solution on the Compressive Strength of PET Bone Scaffolds}

Figure 4 shows that the compressive strength increases as a result of the increasing number of layers, and reaches an optimal $50 \mathrm{~N}$ with the number of layers being 35 . Indicated by the observation made according to Figure 1, PET bone scaffolds composed of a greater number of layers have an orderly arranged structure that provides mutual support between layers and distributes stress evenly, and, therefore, the compact strength is greater. In addition, the PET bone scaffolds are stabilized and bonded by SA solution and the cross-linking by different concentrations of $\mathrm{CaCl}_{2}$ solution, and an increasing concentration results in a tighter fiber arrangement and a more stable structure, which enable the PET bone scaffolds to bear greater compressive strength. 


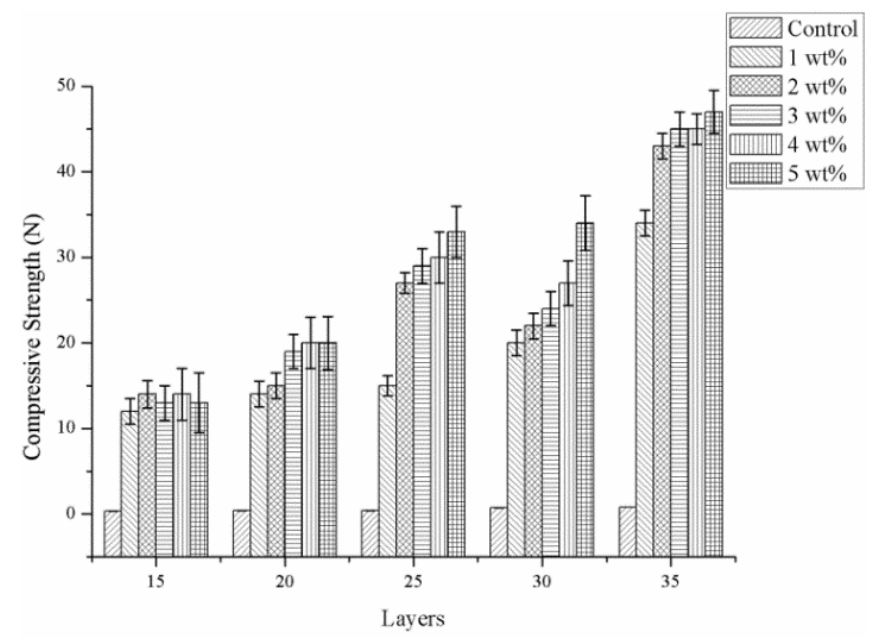

Figure 4. Compressive strength of the PET bone scaffolds composed of various numbers of layers and cross-linked with different concentrations of $\mathrm{CaCl}_{2}$ solution.

\subsection{Effect of Number of Layers and Concentration of $\mathrm{CaCl}_{2}$ Solution on the Morphology of the PET/SA} Bone Scaffolds

Figure 5 shows that the PET filaments are arranged compactly and completely as a result of a high cross-linking level caused by a greater concentration of $\mathrm{CaCl}_{2}$ solution. Therefore, the reticular structure of the SA membranes formed by the interaction of $\mathrm{SA}$ and $\mathrm{CaCl}_{2}$ is firm and not easily damaged. In addition, the hollow PET bone scaffolds that are composed of a greater number of layers can allow the SA membrane to be easily inserted, due to the compact arrangement of filaments. Conversely, the hollow and porous PET bone scaffolds composed of a low number of layers have a loose fiber arrangement, which prevent the SA membranes from being inserted.

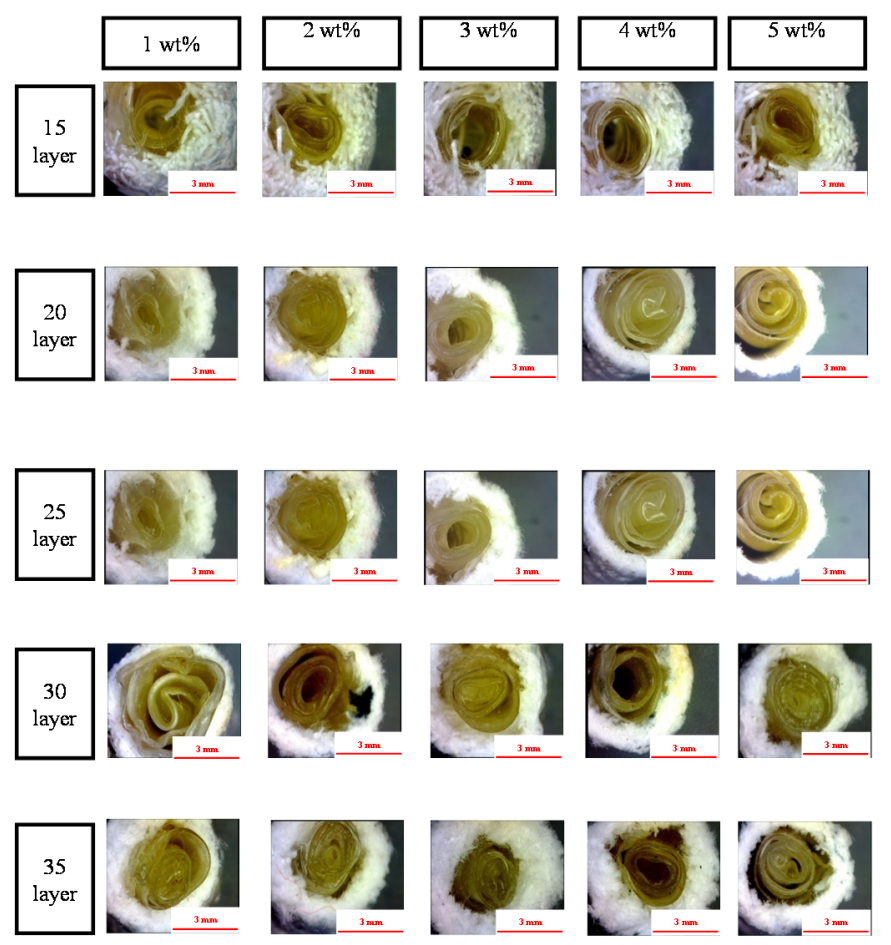

Figure 5. Surface observation of the PET/SA bone scaffolds composed of various numbers of layers and cross-linked with different concentrations of $\mathrm{CaCl}_{2}$ solution. The scale bar is $3 \mathrm{~mm}$. 
2.6. Effect of Number of Layers and Concentration of $\mathrm{CaCl}_{2}$ Solution on the Porosity of the PET/SA Bone Scaffolds

Regarding the application of bone scaffold by braiding, the previous study [7] has shown after implanting braids in the bone defect of rabbits, it can let the tissue infiltrate fiber of braid. Thus, the result has improved the feasibility of cell infiltration. The study is an extension of previous research, and the main purpose is exploring how to let the bone scaffold have porous properties and mechanical properties.

Figure 6 shows that the porosity of the PET/SA bone scaffolds slightly decreases as a result of the increasing number of layers. Namely, the porosity of the PET/SA bone scaffolds is greater when multi-braided with 15 or 20 layers. Comprising fewer layers, the PET/SA bone scaffolds have a loose structure, which decreases the area that the interior of braids and SA membranes adhere, therefore, the porosity is high. A 25-, 30-, or 35-layer braiding can create a compact structure, thereby increasing the adhesion area for the interior of braids and SA membranes. In comparison, a 15- or 20-layer braiding decreases the porosity of the PET/SA bone scaffolds. The porosity of all PET bone scaffolds is between $80 \%$ and $90 \%$ (Figure 2) while the porosity of PET/SA bone scaffolds decreases and is between $60 \%$ and $70 \%$. Such a result is due to the combination of SA membranes, which significantly reduces the interior space of the hollow PET bone scaffolds, and thus the porosity of the PET/SA bone scaffolds decreases.

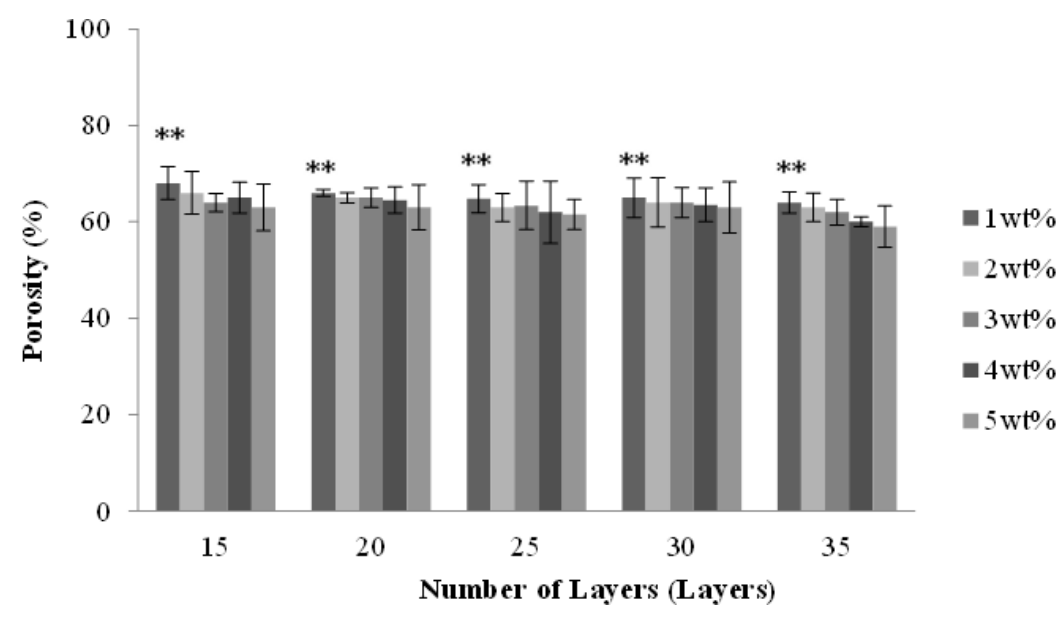

Figure 6. Porosity of the PET/SA bone scaffolds composed of various numbers of layers and cross-linked with different concentrations of $\mathrm{CaCl}_{2}$ solution. ${ }^{* *} p<0.01$.

\subsection{Effect of Number of Layers and Concentration of $\mathrm{CaCl}_{2}$ Solution on the Hydrophilicity of PET/SA Bone Scaffolds}

Figure 7 shows that water contact angle of the PET/SA bone scaffolds is proportional to the number of layers. A greater number of layers results in a smaller pore size of the outer layer (i.e., hollow PET bone scaffold), thereby preventing water molecules from infiltrating the interior, and thus the water contact angle is large. The hollow PET bone scaffolds and PET/SA bone scaffolds have a similar water contact angle, because both have a porous structure and the PET/SA bone scaffolds do not undergo any cross-linking stabilization after the combination of SA membranes. 


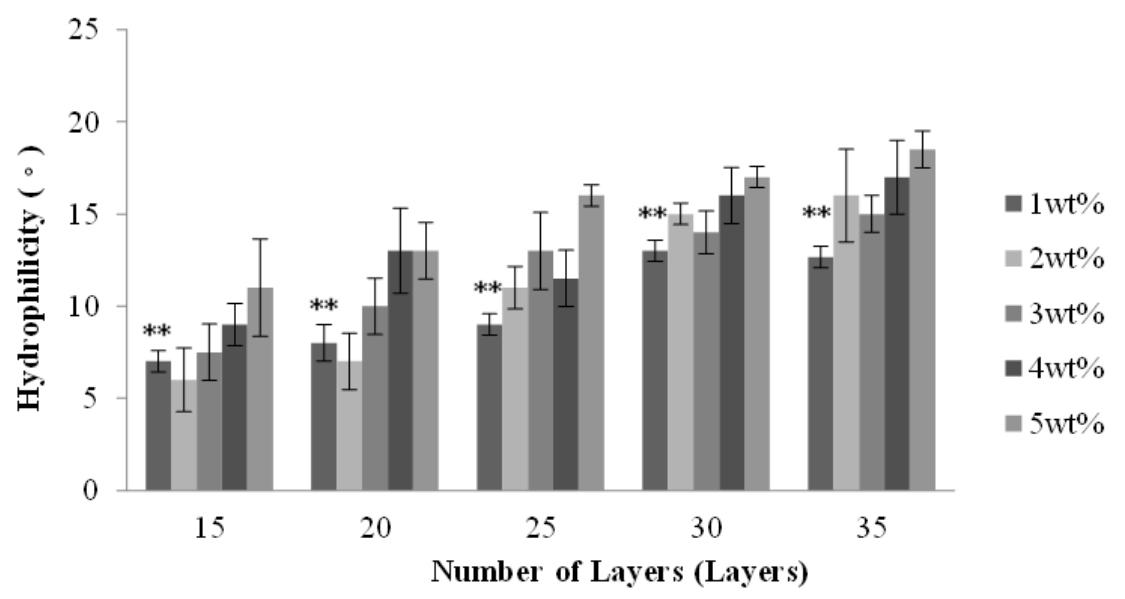

Figure 7. Water contact angle of the PET/SA bone scaffolds composed of various numbers of layers and cross-linked with different concentrations of $\mathrm{CaCl}_{2}$ solution. ${ }^{* *} p<0.01$.

2.8. Effect of Number of Layers and Concentration of $\mathrm{CaCl}_{2}$ Solution on the Compressive Strength of PET/SA Bone Scaffolds

Observed from the cutting sections of PET/SA bone scaffolds, the SA membranes account for the majority of the area, and this area slightly decreases when the PET braids are multi-layer braided with more layers. Namely, the volume of PET/SA bone scaffolds is primarily composed of SA membranes. The compressive load is dissipated by the volume of the samples; therefore, the PET/SA bone scaffolds have a greater compressive strength than PET bone scaffolds, and PET/SA bone scaffolds composed of a high number of layers have a greater compressive strength than those composed of a low number of layers.

Comparing Figures 4 and 8 with a specified number of layers, the PET/SA bone scaffolds have a compressive strength, which is significantly greater than that of hollow PET bone scaffolds. The PET/SA bone scaffolds contain the spiral interlayer of SA membranes, which fortify the resistance of the compressive load of the PET/SA bone scaffolds, subsequently increasing their compressive strength.

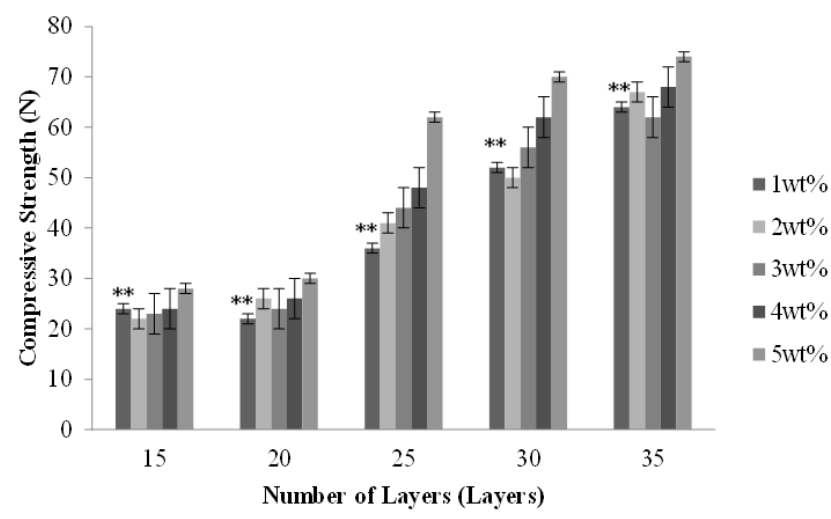

Figure 8. Compressive strength of the PET/SA bone scaffolds composed of various numbers of layers and cross-linked with different concentrations of $\mathrm{CaCl}_{2}$ solution. ${ }^{* *} p<0.01$.

Figure 8 shows that with a 15-layer braiding, the concentrations of $\mathrm{CaCl}_{2}$ are barely correlated with the compressive strength. The PET/SA bone scaffolds that are braided with a low number are constructed with a small thickness, which offers only a small area to bear the compressive load, namely the interlayer takes the majority of the compressive load. The compressive strength of the 
PET/SA bone scaffolds increases with an increase in the number of the layers, as the fibrous layers are neatly and compactly arranged when braided with more layers, thereby enabling the PET/SA bone scaffolds to withstand a greater force. As a result, it is the number of layers, not the concentration of the $\mathrm{CaCl}_{2}$ solution that is highly correlated with the compressive strength of the PET/SA bone scaffolds, determining the former to be a parameter in consideration.

Figure 4 shows that a 15- or 20-layer braiding results in lower compressive strength of the PET/SA bone scaffolds; moreover, a 30- or 35-layer braiding results in a high compressive strength but a low porosity (seen in Figure 6), thereby determining that the optimal number of layers is 25 for the PET/SA bone scaffolds.

According to ASTM standard, the standard of bone substitute is most of the joint categories nowadays. The study develops the bone scaffold, which cures the small bone defect, so it hasn't the standard yet. Thus, the study is based on the related journal of biomaterial, which publishes the research data. According to Torres et al. [8], PET/SA bone scaffolds of the study are highly hydrophilic, and it can conform to the application of bone tissue. On the basis of Karageorgiou et al. [9], when the range of porosity is between $60 \%$ with $70 \%$, it still can do the animal study, and having the osteogenetic properties. In summary, it shows the water contact and porosity of PET/SA bone scaffolds are suitable with implanting. However, compressive strength of study is weaker than Wang et al. [10], but the purpose of study is repairing small segmental bone defects. Compressive strength just protects its porous structure when implanting. Thus, it is enough to implant use in vivo.

\subsection{Cytotoxicity Assay}

Figure 9 shows that the cellular viability after a 24-hour culture with osteoblast cells MG-63 is beyond $100 \%$ for all SA membranes that are cross-linked with various concentrations of $\mathrm{CaCl}_{2}$ solution, and then it reaches $120 \%$ after a 48 -hour culture. Such results are ascribed to the SA hydrogel, which possesses similar bio-mechemical properties to those of extracellular matrix and facilitates the cells' attachment to the polymer networks for proliferation and differentiation [11].

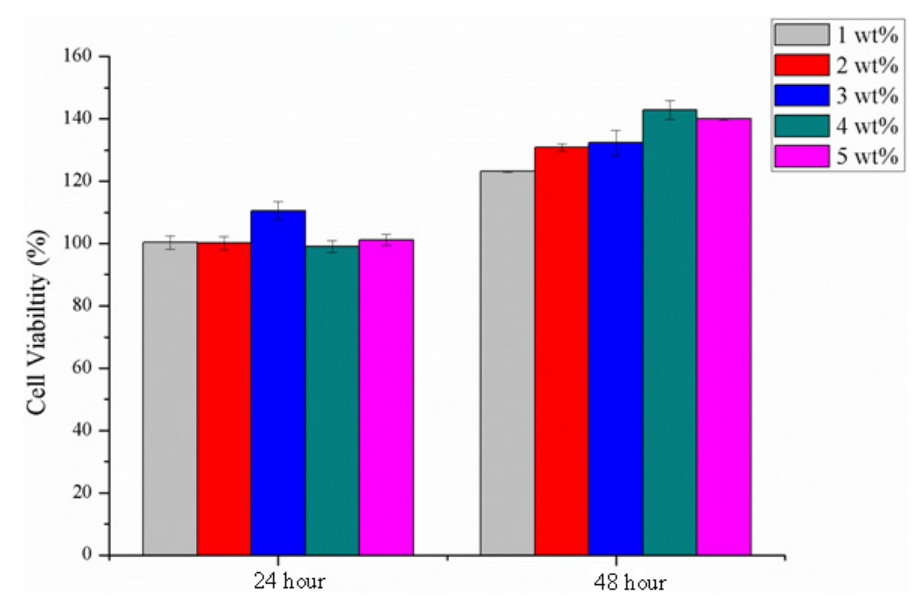

Figure 9. MTT assay of cell viability of the SA membranes as related to various cross-linking concentrations and differing culture spans.

\subsection{Degradation Test}

According to previous reference [7], after a braid is implanted in vivo for about one month, it can allow the tissue infiltration entirely. Figure 10 presents after SA crosslinks with $\mathrm{CaCl}_{2}$, it can degrade to $70 \%$ at least in one month. Thus, it has enough time to allow tissue infiltration. 


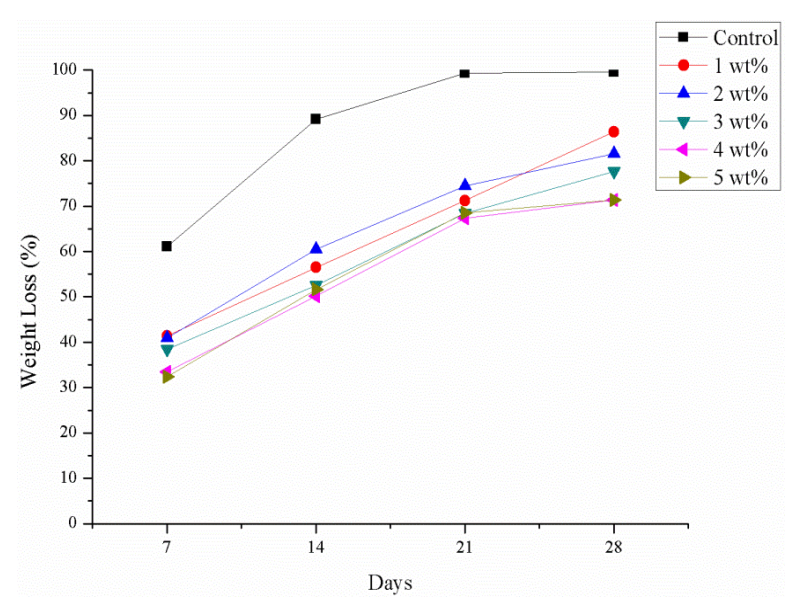

Figure 10. Degradation test.

\section{Experimental}

\subsection{Materials}

Polyethylene terephthalate (PET) filaments (75D/36f) are purchased from Far Eastern New Century, Taiwan. SA (First Chemical Manufacture Co., Ltd., Taipei, Taiwan) has a purity of 96\%. $\mathrm{CaCl}_{2}$ (Choneye Pure Chemicals, Taipei, Taiwan) is at a reagent chemical grade. MTT (3-[4,5-dimethylthiazol-2-yl]-2,5-diphenyltetrazolium bromide) is purchased from Sigma Aldrich, Louis, MO, USA. Dimethyl sulfoxide (DMSO) is purchased from Applichem, Inc., Louis, MO, USA. MG-63 is provided by the Food Industry Development Institute, Hsinchu, Taiwan, and it originates from osteosarcoma of human bone.

\subsection{Preparation of PET Bone Scaffolds}

Two 75 D PET filaments are combined to form 150 D PET plied yarns on a rotor twister with a twist count of seven twists/inch, after which the 150 D PET plied yarns are then coiled onto the carrier. The carrier is then mounted onto a 16-spindle braider (Nan Hsing Machinery \& Co., Ltd., Changhua, Taiwan), where the tooth number of the take-up gear and the braiding gear are specified as 80 and 60 , respectively. During the braiding process, a release paper enwraps a stainless steel mandrel with a diameter of $6 \mathrm{~mm}$ that is affixed in the braiding center. The 150 D PET plied yarns are braided surrounding the mandrel to form 15-, 20-, 25-, 30-, and 35-layer PET porous braids. Afterward, the braids with the mandrel are soaked in a $3 \mathrm{wt} \% \mathrm{SA}$ solution for $30 \mathrm{~min}$ and then cross-linked with a $1,2,3,4$, or $5 \mathrm{wt} \%$ of $\mathrm{CaCl}_{2}$ soluton for $10 \mathrm{~min}$, and dried in an oven. The mandrel is finally removed and the PET braids are trimmed to form the PET porous bone scaffolds.

\subsection{Preparation of $S A$ Membranes}

SA powder and deionized water are formulated into a $3 \mathrm{wt} \% \mathrm{SA}$ solution, and $10 \mathrm{~mL}$ of which is then poured into the dish and dried at $70{ }^{\circ} \mathrm{C}$ to form membranes. The membranes are then respectively cross-linked by $1,2,3,4$, or $5 \mathrm{wt} \% \mathrm{CaCl}_{2}$ solutions, and then dried at $70{ }^{\circ} \mathrm{C}$ again to prepare the SA membranes.

\subsection{Preparation of PET/SA Bone Scaffolds}

SA membranes are immersed in deionized water until they are fully saturated, and then rolled up and immersed into the hollow PET bone scaffolds. The whole structure is dried in an oven at $50{ }^{\circ} \mathrm{C}$ for a setting, forming PET/SA bone scaffolds. 


\subsection{Tests}

\subsubsection{Surface Observation of PET and PET/SA Bone Scaffolds}

Samples are placed on the platform of the stereomicroscope (SMZ-10A, Nikon Instruments Inc., Kōbe-shi, Japan) and analyzed by Motic Images Plus 2.0 software (Motic Group Co., Ltd., Richmond, $\mathrm{BC}$, Canada) in order to determine the influence of cross-linking with different concentrations of $\mathrm{CaCl}_{2}$ solution on the formation of the porous PET bone scaffolds, and observe the appearance of the spiral and porous PET/SA bone scaffolds as well as the combination between the PET bone scaffolds and SA membranes.

\subsubsection{Porosity}

\section{PET Bone Scaffolds}

Samples are cut into $1-\mathrm{cm}$ long pieces and their porosity is calculated with the equation as follows [12]:

$$
\rho_{\mathrm{s}}=\left\{1-\left[\left(m_{\mathrm{s}} /\left(\pi \times\left(R^{2}-d^{2}\right) \times l\right)\right] / \rho_{\mathrm{i}}\right\} \times 100 \%\right.
$$

where $\rho_{\mathrm{s}}$ is the real porosity, $m_{\mathrm{s}}$ is the sample weight, $R$ is the outer diameter of the sample, $d$ is the inner diameter of the sample, $l$ is the length of the sample, and $\rho_{\mathrm{i}}$ is the specific density of the sample $\left(1.24 \mathrm{~g} / \mathrm{cm}^{3}\right)$.

\section{PET/SA Bone Scaffolds}

Samples are measured for their radius and height, which are used to calculate the total volume $\left(V_{\mathrm{t}}\right)$ with Equation (8). Samples are immersed in the deionized water in order to measure their real volume $\left(V_{\mathrm{r}}\right)$ and the equation calculates the porosity of the samples.

$$
\left(V_{\mathrm{t}}-V_{\mathrm{r}}\right) / V_{\mathrm{t}} \times 100 \%
$$

\subsubsection{Hydrophilicity of PET and PET/SA Bone Scaffolds}

Samples are cut into $1 \mathrm{~cm}$ long pieces and measured by a water contact angle tester (CA-D, Kyowa Interface Science Co., Ltd., Saitama, Japan) for the water contact angle.

\subsubsection{Compressive Strength of PET and PET/SA Bone Scaffolds}

This test follows ASTM D6641/D6641M-09 [13] with a compressive speed being $1.3 \mathrm{~mm} / \mathrm{min}$ and a distance between clamps being $1.5 \mathrm{~cm}$. Samples are cylinder and first attached to the clamp plane to be upright for the compressive strength test.

\subsubsection{Cytotoxicity Assay}

After being sterilized by $12 \mathrm{kGy} \gamma$-ray, SA membranes are measured for their cellular viability (\%) by MTT assay as specified in ISO 10993-5 [14]. SA membranes are then soaked in medium to form the extract at $0.45 \mathrm{mg} / \mathrm{cc} 10 \mu \mathrm{L}$ of sample extract is seeded into a 96-well culture plate, followed by the addition of $90 \mu \mathrm{L}$ of osteoblast cells MG-63 suspensoin with a density of $10^{4}$ cell/well. The culture plate is then placed in an incubator for 24 and $48 \mathrm{~h}$, and then moved to a horizontal laminar flow table where the medium is removed by a Pasteur pipette. Then, $70 \mu \mathrm{L}$ of the mixture of MTT and medium (1:39) is added, and the culture plate is kept in darkness for $4 \mathrm{~h}$. After the mixture is removed and $70 \mu \mathrm{L}$ DMSO solvent is added, an ELISA reader (Feng Jih Biomedical \& Instruments Co., Ltd., Taipei, Taiwan) measures the absorbance of the sample extract at $570 \mathrm{~nm}$. The yielded optical density (OD) is then used for the calculation of cellular viability (\%) with the following equation:

$$
\text { Cellular viability }(\%)=(\text { medium containing the extract solution optical densities }
$$

$$
\text { of/optical densities of the fresh medium) } \times 100 \%
$$


According to the Figure 11, it shows that after sterilizing, functional groups of SA do not have any change. The process doesn't change film properties such as stretching vibrations of $\mathrm{O}-\mathrm{H}$ bond $\left(3200 \mathrm{~cm}^{-1}\right)$ aliphatic C-H $\left(2927-2850 \mathrm{~cm}^{-1}\right)$ [15].

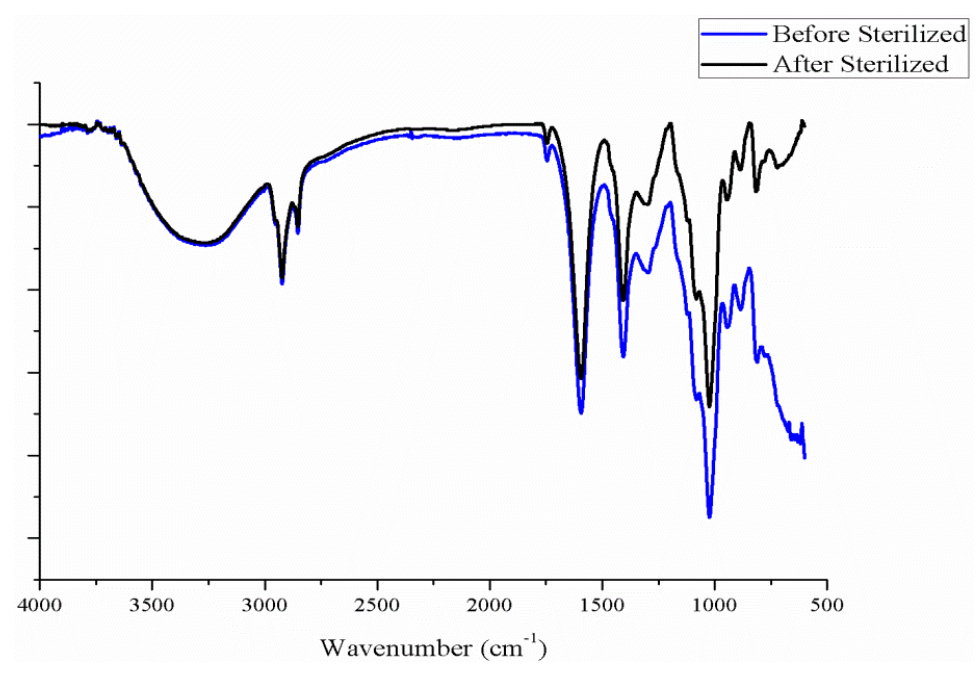

Figure 11. Fourier-transform infrared spectroscopy (FT-IR) spectra of the sodium alginate.

\subsubsection{Degradation Test}

Samples are sterilized by $12 \mathrm{kGy} \gamma$-ray, weighed $\left(W_{0}\right)$, and placed in a centrifugal tube containing phosphate buffered saline (PBS), and the whole structure is then placed in a water bath with shaker for seven, 14,21 or 28 days. Samples are then removed, dried at $60^{\circ} \mathrm{C}$, and then weighed $\left(W_{\mathrm{t}}\right)$. The collected data is then calculated by the following equation:

$$
\text { Weight Loss }(\%)=\left(W_{0}-W_{\mathrm{t}}\right) / W_{\mathrm{t}} \times 100 \%
$$

\subsubsection{Statistical Analysis}

All results were expressed as means \pm standard deviation (SD) $(n \geqslant 5)$. Data were analyzed using one-way analysis of variance (ANOVA). $\left({ }^{*}\right)$ indicates a significant difference in comparison to other value, $p<0.05 ;\left(^{* *}\right)$ indicates a significant difference in comparison to other value, $p<0.01$.

\section{Conclusions}

This study successfully produces the PET/SA bone scaffolds. Using a braiding technique, PET filaments are made into the porous outer layer of the bone scaffolds for the support to the load, while SA membranes serve as the interlayer, which simulate the extracellular matrix for cell attachment and proliferation. When composed of a greater number of layers, the structure of the PET braids becomes compact instead of loose; therefore, the immersion in SA solution and the cross-linking with $\mathrm{CaCl}_{2}$ solution stabilize the braiding structure and also make PET filaments more tightly arranged. With an increase in the number of layers and an increase in the concentration of $\mathrm{CaCl}_{2}$ solution, the resulting hollow PET bone scaffolds have a porosity, which decreases by $11 \%$ while their compressive strength increases by $80 \%$.

Compared to hollow PET bone scaffolds, the PET/SA bone scaffolds also have a significantly lower porosity and greater compressive strength. The MTT assay results show that the cell viability of all SA membranes remains beyond $100 \%$ after a 48 -hour culture, indicating that the cross-linking with $\mathrm{CaCl}_{2}$ solution does not influence the good cell viability of $\mathrm{SA}$ membranes.

Acknowledgments: The authors would especially like to thank the National Science Council of the Republic of China, Taiwan, for financially supporting this research under Contract NSC 101-2622-E-039-001-CC3. 
Author Contributions: In this study, the concepts and designs for the experiment, all required materials, as well as processing and assessment instrument are provided by Jia-Horng Lin and Ching-Wen Lou. Data are analyzed, and experimental results are examined by Chien-Lin Huang, Chi-Fan Liu, and Chih-Kuang Chen. The experiment is conducted and the text is composed by Shih-Peng Wen.

Conflicts of Interest: The authors declare no conflict of interest.

\section{References}

1. Valente, J.F.A.; Valente, T.A.M.; Alves, P.; Ferreira, P.; Silva, A.; Correia, I.J. Alginate based scaffolds for bone tissue engineering. Mater. Sci. Eng. C 2012, 32, 2596-2603. [CrossRef]

2. Aflori, M.; Drobota, M.; Dimitriu, D.G.; Stoica, I.; Simionescu, B.; Harabagiu, V. Collagen immobilization on polyethylene terephthalate surface after helium plasma treatment. Mater. Sci. Eng. B 2013, 178, 1303-1310. [CrossRef]

3. Saito, T.; Takemoto, M.; Fukuda, A.; Kuroda, Y.; Fujibayashi, S.; Neo, M.; Honjoh, D.; Hiraide, T.; Kizuki, T.; Kokubo, T.; et al. Effect of titania-based surface modification of polyethylene terephthalate on bone-implant bonding and pen-implant tissue reaction. Acta Biomater. 2011, 7, 1558-1569. [CrossRef] [PubMed]

4. Rani, V.V.D.; Ramachandran, R.; Chennazhi, K.P.; Tamura, H.; Nair, S.V.; Jayakumar, R. Fabrication of alginate/nano $\mathrm{TiO}_{2}$ needle composite scaffolds for tissue engineering applications. Carbohydr. Polym. 2011, 83, 858-864. [CrossRef]

5. Morais, D.S.; Rodrigues, M.A.; Silva, T.I.; Lopes, M.A.; Santos, M.; Santos, J.D.; Botelho, C.M. Development and characterization of novel alginate-based hydrogels as vehicles for bone substitutes. Carbohydr. Polym. 2013, 95, 134-142. [CrossRef] [PubMed]

6. Liu, Y.C.; Lim, J.; Teoh, S.H. Review: Development of clinically relevant scaffolds for vascularised bone tissue engineering. Biotechnol. Adv. 2013, 31, 688-705. [CrossRef] [PubMed]

7. Lou, C.W.; Yao, C.H.; Chen, Y.S.; Lu, C.T.; Chen, W.C.; Yen, K.C.; Lin, J.H. PLA/ $\beta-T C P$ complex tubes: The mechanical properties and applications of artificial bone. J. Biomater. Sci. Polym. 2012, 23, 1701-1712. [CrossRef] [PubMed]

8. Torres, A.L.; Gaspar, V.M.; Serra, I.R.; Diogo, G.S.; Fradique, R.; Silva, A.P.; Correia, I.J. Bioactive polymeric-ceramic hybrid 3D scaffold for application in bone tissue regeneration. Mater. Sci. Eng. C 2013, 33, 4460-4469. [CrossRef] [PubMed]

9. Karageorgiou, V.; Kaplan, D. Porosity of 3D biomaterial scaffolds and osteogenesis. Biomaterials 2005, 26, 5474-5491. [CrossRef] [PubMed]

10. Wang, J.; Yu, X. Preparation, characterization and in vitro analysis of novel structured nanofibrous scaffolds for bone tissue engineering. Acta Biomater. 2010, 6, 3004-3012. [CrossRef] [PubMed]

11. Langer, R.; Vacanti, J.P. Tissue engineering. Science 1993, 260, 920-926. [CrossRef] [PubMed]

12. Chung, S.; Ingle, N.P.; Montero, G.A.; Kim, S.H.; King, M.W. Bioresorbable elastomeric vascular tissue engineering scaffolds via melt spinning and electrospinning. Acta Biomater. 2010, 6, 1958-1967. [CrossRef] [PubMed]

13. American Society for Testing Materials. Standard Test Method for Compressive Properties of Polymer Matrix Composite Materials Using a Combined Loading Compression (CLC) Test Fixture; ASTM D6641/D6641M-09; ASTM International: West Conshohocken, PA, USA, 2009.

14. International Organization for Standardization. Biological Evaluation of Medical Devices-Part 5: Tests for in vitro Cytotoxicity; ISO 10993-5:2009; International Organization for Standardization: Geneva, Switzerland, 2009.

15. Daemi, H.; Barikani, M. Synthesis and characterization of calcium alginate nanoparticles, sodium homopolymannuronate salt and its calcium nanoparticles. Sci. Iran. 2012, 19, 2023-2028. [CrossRef]

(C) 2015 by the authors; licensee MDPI, Basel, Switzerland. This article is an open access article distributed under the terms and conditions of the Creative Commons by Attribution (CC-BY) license (http:/ / creativecommons.org/licenses/by/4.0/). 